$\xi=-1$

\title{
A study on the solid image projection mapping system in the curved display of the face shape for the performance art
}

\author{
Eun Seo Song ${ }^{1}$, Gil Sang Yoo ${ }^{2}$, Sung-Dae Hong ${ }^{3 *}$ \\ ${ }^{I}$ Department of Flim and Digital Media Center,Seokyeing University, Seokyeong 702. Hyein-Bld. 124 Seokyeong-Ro Singbuk-Ku Seoul, \\ 02713, Seoul, Republic of Korea \\ ${ }^{2}$ Department of Computer Science and Engineering, Korea University, 145 Anam-ro, Seongbuk-gu, Seoul, 20841, Republic of Korea \\ ${ }^{3}$ Professor, Dept. of Film and Digital Media, Seokyeong University t, 124 Seogyeong-ro, Seongbuk-gu, Seoul, Republic of Korea, 02713 \\ *Corresponding author E-mail: sungdaehong@gmail.com
}

\begin{abstract}
Background/Objectives: Aims to study the projection mapping technology which will project the multi-phase solid image in the curved display real time in the performance.

Methods/Statistical analysis: Analyze the shape of the object to be mapped for the project and based on the analyzed characteristics, the structure of the installation of the applicable project to the actual object and the projection mapping production tool shall be developed and be applied to the elastic shape.

Findings: According to the analysis result of the curved display, one projector did not suit the image and the distortion of the image occurred. According, it was divided into the four sections and projection mapping was made to minimize the problems, and the projection production tool which applied the geometric matching technology, edge blending technology, UDP telecommunication method etc based on the grid for the image matching using the Max/Msp was developed and the actual face shaped curved display was applied.

Improvements/Applications: In order to conduct projection map precisely to the actuator moving real time, Z-depth should be considered and the advanced technology which matches the three dimensional mapping image should be applied.
\end{abstract}

Keywords: Projection Mappin; Curved Display; Max/MSP; Edge Blending; Warping.

\section{Introduction}

Recently, as the digital technology develops rapidly, the 2D image has been changed to $3 \mathrm{D}$ image gradually and in order to express the solid immersion feeling of the 3D image, the projection mapping technology is being applied to various contents. Projection mapping is the technology which projects the solid image to the object and maximizes the solid feeling and in the beginning it was used mainly in buildings to map the solid image, but recently it is also being used in the exhibition, fashion show, advertisement and performances etc. [1]

The modern performance is making a new challenge fused with the cutting-edge technologies which escapes from the conventional analogue emotions. As a scheme to maximize the visual effect expressed in the performance, the optical illusion effect is shown by projection mapping the solid image.

The shape of the projection mapping used mainly in the performances projects the solid image in the plane screen and shows the spatial expandability. The projection mapping technology was applied to the moving objects, people's face, body and clothes, going beyond the conventional method and was tried.

This thesis intends to study the projection mapping technology which can project multi-facet solid image to the curved display real time in the performance. It shall analyze the characteristics of the shape of the object to which the solid image will be projected and based on the analyzed characteristics, the technical solution scheme which can be applied to the actual object is deducted and the real time multi-facet projection mapping system for the dynamic object objects with elastic shape is proposed.

\section{Projection mapping}

Projection mapping, also known as video mapping and spatial augmented reality, is a projection technology used to turn objects, often irregularly shaped, into a display surface for video projection. Technology used to turn objects, often irregularly shaped, into a display surface for video projection. The projection mapping is the technology which analyzes the 3 dimensional characteristics of a specific object and projects the solid image and expresses the optical illusion effect visually of specific objects and 3 dimensional images. Since the projection mapping pursues the natural reinforcement, and thus the cases that the graphic images go beyond the screen scope should be minimized and should be realized using a commercial program such as the Mad Mapper $^{2}$.Since the projection mapping projects the virtual images to the dark space and expresses the marvelous fantasy, the views of the audience can be concentrated and thus is being used in media façade, exhibition, performance and advertisement field etc. [3].

\subsection{Mapping cases of the three dimensional objects}

The three dimensional objects need the analysis of the shape, size, texture, color and movement of the objects one dimensionally. When the analysis will be finished, the technologies which can be applied to the objects shall be deducted and the optimal virtual image can be expressed. [4]

The ShoyoMujo which was manufactured in 2014 mapped the dynamic graphic to the skull shape. [4] projectors were installed 
on each corner in the square structure in order to map the 12 feet size of skull shaped model and the graphic images were all projected on the two skulls. By modeling the correct modeling using a three dimensional program, results can be predicted and thus more precise images were realized in figure [1-5].

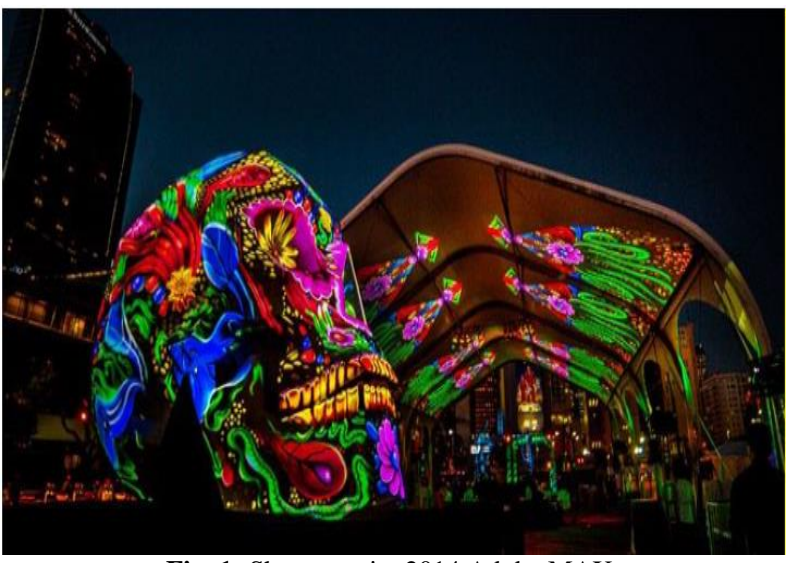

Fig. 1:.Shogyomujo, 2014 Adobe MAX.

By applying the projection mapping to the clothes of the actors in the performance, Turando, the solid clothes were described and the clothes expression scope has been expanded. 4 projectors were used to realize without the square regions in the clothes of the actors and by connecting the Kinect, Zigfu, Unitiy three D programs, the interactive projection mapping tool has been developed and the projection mapping technology which pursues the movement of the actor was used in figure [2-6].
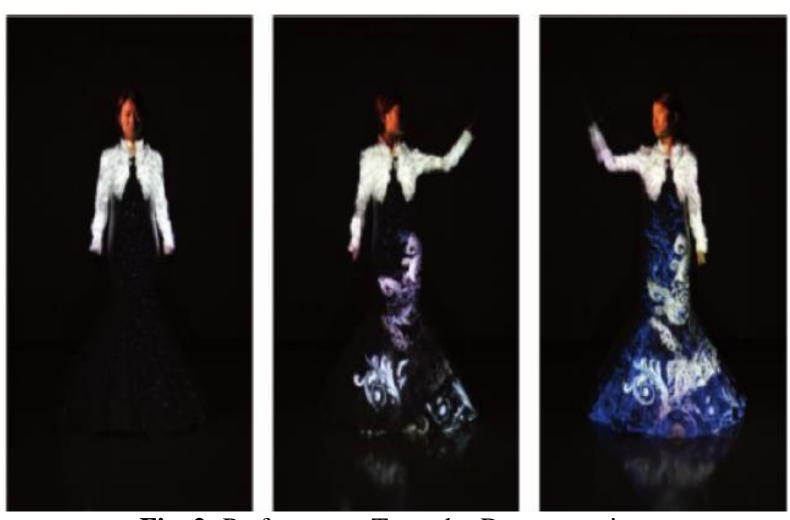

Fig. 2:.Performance Turandot Demonstration.

\section{Projection mapping realization method of dynamic display}

\subsection{Curved projection mapping structure}

The curved projection mapping should search for the optimal method to realize without the square zone when projecting the solid images to the object by analyzing the shape of the objects. The curved display <Figure 3> can hardly project the whole display with one side projection by realizing the shape of semihemisphere. Thus, a structure which is suitable for the curved display shale was set. Shape of the semi-hemisphere has been divided into four facets and projected and thus although the audience watch from various angles, realization of images unified without the square zone is possible in Figure 3.
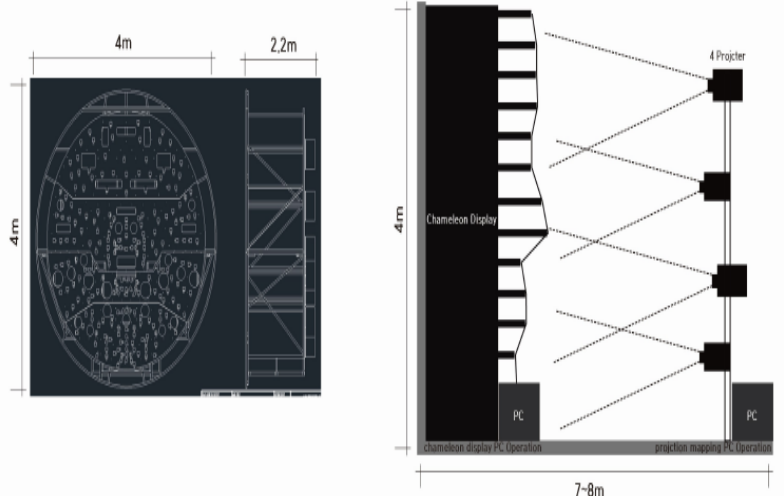

Fig. 3: Display Installation.

\subsection{Curved projection mapping program}

Since the multi-facet projection mapping manufacturing tool needs to be developed in order to project the solid images on the curved display, this paper has analyzed the interface of the commercialized projection mapping program used in the performance and exhibitions as shown in table [1-7].

Based on the analysis of the interface of the above commercial program, necessary elements needed for the multi-facet mapping production tool were added and the production tool was designed in figure 4.

Table 1: Projection Mapping Program Analysis

\begin{tabular}{|c|c|c|c|c|c|c|c|}
\hline Function/program & Mad Mapper & Module8 & HeavyM & Touch designer & VVVV & ISADORA & Pandoras Box \\
\hline Service Environment & Mac & Mac & Win/Mac & Win/Mac & Win & Win/Mac & Win/Mac \\
\hline Real-time Control & $\mathrm{Y}$ & $\mathrm{Y}$ & $\mathrm{Y}$ & $\mathrm{Y}$ & $\mathrm{N}$ & $\mathrm{Y}$ & $\mathrm{Y}$ \\
\hline Preview & $\mathrm{Y}$ & $\mathrm{Y}$ & $\mathrm{Y}$ & $\mathrm{Y}$ & $\mathrm{Y}$ & $\mathrm{Y}$ & $\mathrm{Y}$ \\
\hline Preview of many sides & $\mathrm{Y}$ & $\mathrm{Y}$ & $\mathrm{N}$ & $\mathrm{Y}$ & $\mathrm{Y}$ & Y & $\mathrm{Y}$ \\
\hline Play mode & $\mathrm{Y}$ & $\mathrm{Y}$ & $\mathrm{Y}$ & $\mathrm{Y}$ & $\mathrm{Y}$ & Y & $\mathrm{Y}$ \\
\hline Masking & $\mathrm{Y}$ & $\mathrm{Y}$ & $\mathrm{Y}$ & $\mathrm{Y}$ & $\mathrm{Y}$ & Y & $\mathrm{Y}$ \\
\hline Mesh Warping & $\mathrm{Y}$ & $\mathrm{N}$ & $\mathrm{N}$ & $\mathrm{Y}$ & $\mathrm{Y}$ & $\mathrm{Y}$ & $\mathrm{Y}$ \\
\hline Bezier Curve & $\mathrm{Y}$ & $\mathrm{N}$ & $\mathrm{N}$ & $\mathrm{Y}$ & $\mathrm{Y}$ & $\mathrm{N}$ & $\mathrm{Y}$ \\
\hline 2D Mesh & $\mathrm{Y}$ & $\mathrm{N}$ & $\mathrm{N}$ & $\mathrm{Y}$ & $\mathrm{Y}$ & $\mathrm{Y}$ & $\mathrm{Y}$ \\
\hline 3D Mesh & $\mathrm{Y}$ & $\mathrm{N}$ & $\mathrm{N}$ & $\mathrm{Y}$ & $\mathrm{Y}$ & Y & $\mathrm{Y}$ \\
\hline Soft edge & $\mathrm{Y}$ & $\mathrm{N}$ & $\mathrm{Y}$ & $\mathrm{Y}$ & $\mathrm{Y}$ & $\mathrm{N}$ & $\mathrm{Y}$ \\
\hline Blend mode & $\mathrm{Y}$ & $\mathrm{Y}$ & $\mathrm{Y}$ & $\mathrm{Y}$ & $\mathrm{Y}$ & $\mathrm{N}$ & $\mathrm{Y}$ \\
\hline Figure/lines(textured line and animation) & $\mathrm{Y}$ & $\mathrm{N}$ & $\mathrm{Y}$ & $\mathrm{Y}$ & $\mathrm{Y}$ & $\mathrm{Y}$ & $\mathrm{Y}$ \\
\hline Keystone & $\mathrm{Y}$ & $\mathrm{Y}$ & $\mathrm{Y}$ & $\mathrm{Y}$ & $\mathrm{N}$ & $\mathrm{Y}$ & $\mathrm{Y}$ \\
\hline Multi-Image input/output & $\mathrm{Y}$ & $\mathrm{Y}$ & $\mathrm{N}$ & $\mathrm{Y}$ & $\mathrm{Y}$ & Y & $\mathrm{Y}$ \\
\hline Remote Communications & $\mathrm{Y}$ & $\mathrm{N}$ & $\mathrm{N}$ & $\mathrm{N}$ & $\mathrm{N}$ & $\mathrm{N}$ & $\mathrm{Y}$ \\
\hline
\end{tabular}




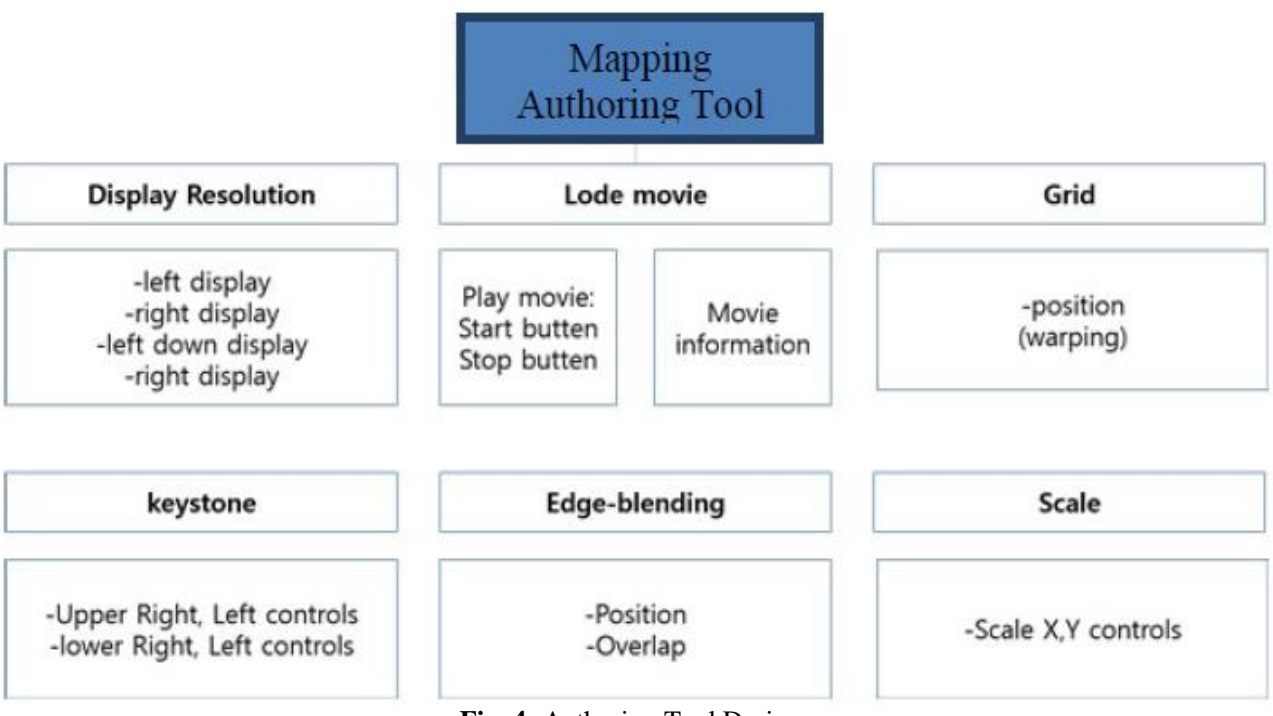

Fig. 4: Authoring Tool Design.

\subsection{Real time multi image matching technology}

In order to match the multi images real time, the grid-based geometric matching technology, up and down and right and left edge blending technology based on multi facets are needed. Also, in order to reduce the spatial limitations, the remote telecommunications method was applied and by using the Mas/Msp, the method of connecting the above objects one another (OOP: Object oriented program), the projection mapping production tool has been developed. [8].

\subsubsection{Multi projection mapping image monitoring}

Since the window of the preview is composed of [4] each maximum, images can be extracted from the four projects. Separate name folder for each name can be designated for the image source to be executed in the production tool and be dragged and be contained in the server. Also, it was designed in such a way that play becomes possible by maintaining the 24 pfs speed continuously. In Figure 5, preview screen has been developed so that the manager can monitor the projection mapping image and was designed in such a way that repetitive play cannot be made after the mapping image is executed.

\section{Multi Source Monitoring}

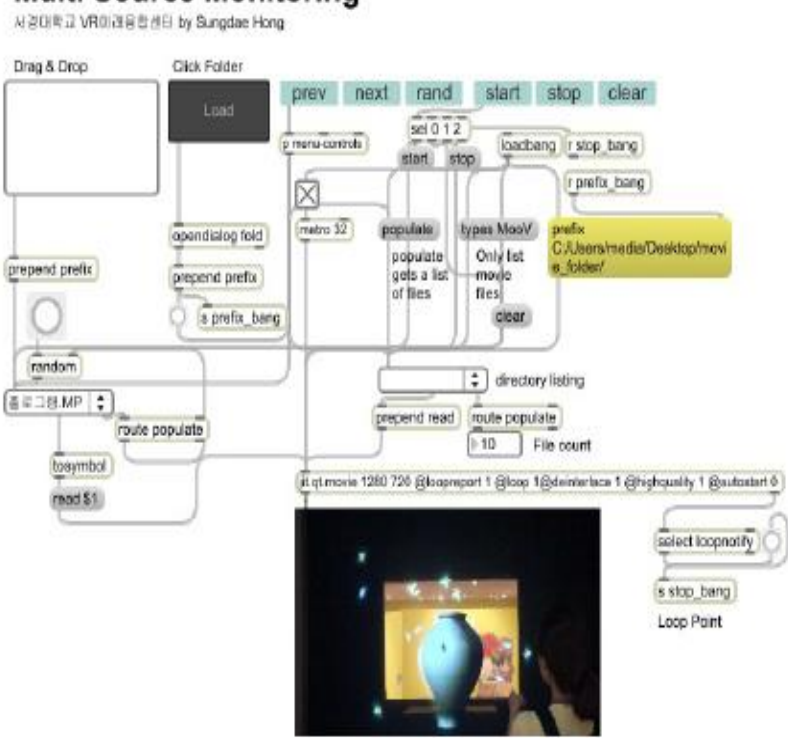

Fig. 5:.Multi Source Monitoring.

\subsubsection{Multi image matching technology}

In order to match multi images, the edge blending, keystone adjustment and multi sources etc based on the four facets were designed and based on these four facets, the texture off-set of the input image has been designed and was applied to the production tool. By considering the performance of the [4] faceted projection mapping controller and by applying the hap codes plug-ins, it was applied so that the real time process speed shall be over $24 \mathrm{fps}$ as the type of reducing minimally the load of the CPU and the CPU of the computer and the control function has been approved and was applied. [9].

The grid-based geo-metric matching technology is needed to match the image so that the multi-image can be shown in one unified image. If you project the image on the curved display, image distortion can occur depending on the degree of the roundness, thus, by applying the grid-based wrapping technology, if you change the location of the pixel, the distortion of the image projected through the projection can be reduced.

In figure 6 , the edge blending function has been applied to match the image. Since the unified image can only be expressed when all the four facets are edge blended, the edge blending function has been applied to all the frames of the images and by adjusting the light and shade and gamma value in the overlapped scope part, the matching of the images was made and was shown to be one unified image [9-10].

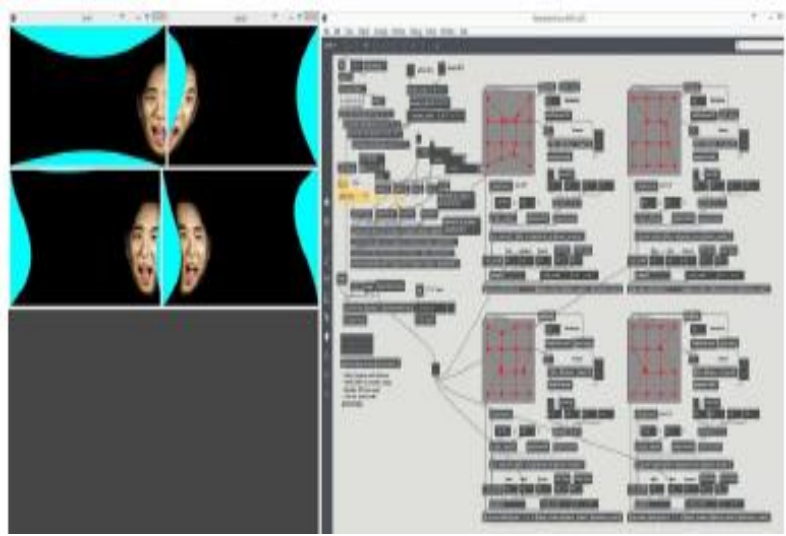

Fig. 6: Multi Image Matching Technology.

\subsubsection{Remote telecommunication}

When installing the curved display which was multi facet projection mapped in the performance, since the environment of the performance halls differ, installation problems can occur in some performance halls due to the environmental limitations. ${ }^{9}$ Accord- 
ingly, in order to reduce the environmental limitations, if we use the remote telecommunication technology which can process the information through the telecommunications signals among the scattered computers in remote areas with the computer, the manager can more easily match the image in figure 7 .

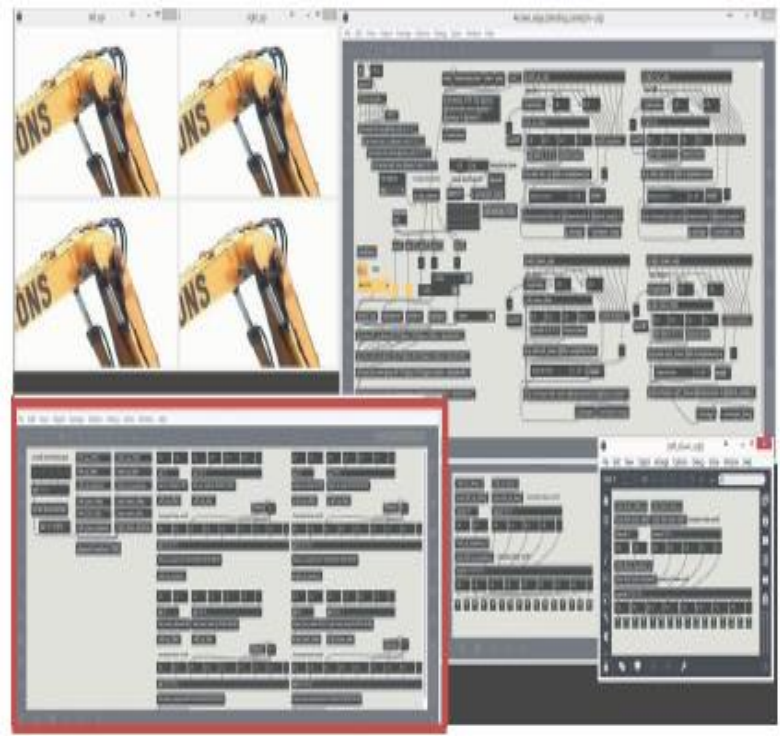

Fig. 7: UDP Remote Telecommunication.

\section{Conclusion}

This study was a study to deduct the basic development data in order to develop the multi-facet projection mapping system real time on the curved display. In order to reduce the distortion of the images in the display of face type, images were projected and the multi-facet projection mapping production tool which match 4 faceted projected images into ne unified image was developed.

In this project, a new form of dynamic display was developed by designing a linear actuator module, developing driving and control technology to create a moving surface, attaching a skin to act as a screen on the actuator surface, creating a curved surface, and then projecting solid images on the skin through projection mapping of dynamic multi-source image in figure 8 .

In the future studies, if you wish to project map precisely on the moving actuator real time, the Z-depth should be considered and the advanced technology to match the [3] dimensional mapping images will have to be applied. Thus, studies on the in-depth production tool to project solid images on the display with constant movement should be proceeded with.

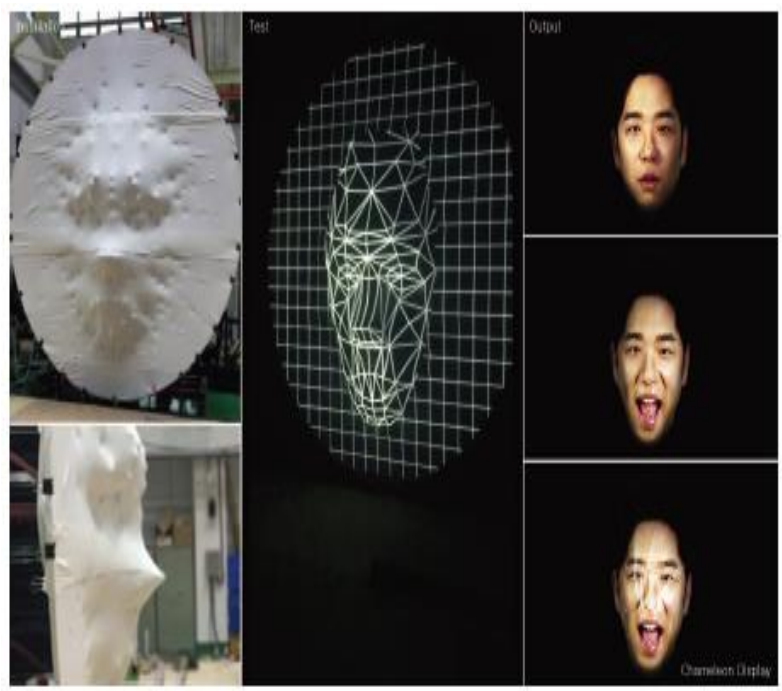

Fig. 8: Surface Display Projection Mapping Results.

\section{Acknowledgment}

This research is supported by Ministry of Culture Sports and Tourism (MCST) and Korea Creative Content Agency (KOCCA) in the Culture Technology (CT) Research \& Development Program 2017.

\section{References}

[1] Jaewoon Lee, Yeonjin Kim, Dongho Kim, Realtime Projection Mapping on Flexible Dynamic Objects, the HCI Society of Korea, 2012, pp. 197-190.

[2] https://en.wikipedia.org/wiki/Projection_mapping.

[3] Yoon-Jung, Lee, A Study on Projection Mapping for Brand Promotion, Konyang University, Dept. of Visual Design, 2013, 16(1) pp.185-193.

[4] Gapwan Hwang, Hyunjhin Lee, Gaphwan Hwang, A Research on Depth Perception caused by Attributes of Projection Mapping on Physical Space, KOREA DIGITAL DESIGN COUNCIL, 2010, 10(4), 253-262.

[5] http://create.adobe.com/2014/11/25/art_on_the_p laya shogyo mujo.html.

[6] Byoung Sue Kang, Jae Young Kim, Jung Hwan Sung, Design and Production of Transforming Dress -Focused on the Morphological Transformation-, Design Convergence Study, 2012, 35(11) no.4, pp. 59-70.

[7] http://madmapper.com/madmapper/.

[8] http://vdmx.vidvox.net/tutorials/guest-tutorial-using-vdmx-withblendy-vj.

[9] http://vdmx.vidvox.net/blog/hap.

[10] Hye Sook Lee, A Study on Distance Education Using Computer Communication Learning System, EwhaWomans University graduate school education engineering MA, 1991, p. 31.

[11] http://m.blog.daum.net/ru1312/158. 